\title{
ASYMPTOTIC STABILITY OF FUNCTIONAL EQUATIONS BY FIXED POINT THEOREMS
}

\author{
MUHAMMAD N. ISLAM ${ }^{1}$ AND JEFFREY T. NEUGEBAUER ${ }^{2}$ \\ ${ }^{1}$ Department of Mathematics \\ University of Dayton \\ Dayton, OH 45469, USA \\ ${ }^{2}$ Department of Mathematics and Statistics \\ Eastern Kentucky University \\ Richmond, KY 40475, USA
}

\begin{abstract}
For many years, Liapunov's direct method has been the primary technique for studying various stability also known as 'Liapunov stability' of functional differential equations. Recently, it has been noticed that some difficulties can arise when Liapunov's method is applied to certain equations, and that a suitable fixed point theorem can overcome some of these difficulties. In this paper we study a particular stability which differs from the Liapunov stability. In particular, we study the existence of asymptotically stable solutions of a system of nonlinear Volterra integral equations. We employ a fixed point theorem due to Krasnosel'skii in the analysis.
\end{abstract}

AMS Subject Classification: $47 \mathrm{H} 10$

Received: June 13, 2017; $\quad$ Accepted: October 31, 2018 ;

Published: November 13, 2018 doi: 10.12732/caa.v22i4.8

Dynamic Publishers, Inc., Acad. Publishers, Ltd. http://www.acadsol.eu/caa 


\section{INTRODUCTION}

For over a half century, researchers have employed Liapunov's direct method to study various types of stability (also known as Liapunov stability) of functional differential equations. Lately, it has been found that for certain equations, Liapunov's method may not be the most convenient method to study these stabilities. This is mainly due to the difficulties of constructions of appropriate Liapunov functions, which are required to study the stability properties of these equations. To overcome this difficulty, one might be able to employ a fixed point theorem and obtain the desired stability property. A good source on this topic is the book by Burton [5].

In this paper, we study a different type of stability, given below in Definition 1.1, which is found in $[1,2,4]$. In particular, we study the existence of asymptotically stable solutions of the following system of nonlinear Volterra integral equations

$$
x(t)=f(t, x(t))+\int_{0}^{t} K(t, s) g(s, x(s)) d s,
$$

where $f, g: \mathbb{R}_{+} \times \mathbb{R}^{n} \rightarrow \mathbb{R}^{n}$ are continuous functions, and $K(t, s)$ is a continuous $n \times n$ matrix function on $0 \leq s \leq t<\infty$. We employ a fixed point theorem due to Krasnosel'skii in the analysis. The readers familiar with Liapunov stability will find that the stability defined in Definition 1.1 is quite different than the standard definition of the Liapunov stability.

We made no effort to study the stability of (1.1) by Liapunov's method. Interested readers familiar with Liapunov stability may find the outcome of our work in this paper to be useful, and they may try to study this type of stability employing Liapunov's method. Studies of that type can then reveal information as to whether there are difficulties associated with Liapunov's method and whether the use of a fixed point theorem can overcome these difficulties for this type of integral equations.

We refer the readers to the this partial list $[3,6,7,8,9,11,12,13]$ for various studies of Liapunov stability of differential and integral equations. This list is certainly neither complete nor comprehensive.

To explain the difficulties associated with Liapunov's method and then how to overcome these difficulties by the use of a fixed point theorem, we 
consider a delay differential equation as an example, and study the asymptotic stability (or Liapunov asymptotic stability) of the zero solution employing Liapunov's method. Then we use contraction mapping principle to show how that difficulty can be avoided. Consider

$$
x^{\prime}(t)=-a x(t)+b x(t-r),
$$

where $a, b$ and $r$ are constants, $r \geq 0$. If $a>0$ and $|b|<a$, then the zero solution of (1.2) is asymptotically stable for every $r \geq 0$.

This can be proven using Liapunov's method or by employing the Banach fixed point theorem. First, define a Liapunov function by

$$
V\left(x_{t}\right)=x^{2}(t)+|b| \int_{t-r}^{t} x^{2}(s) d s .
$$

Then the derivative of $V$ along an (unknown) solution of (1.2) satisfies

$$
V^{\prime}\left(x_{t}\right) \leq-k x^{2}
$$

where $k=-2 a+2|b|$. Also, notice

$$
|x(t)|^{2} \leq V\left(x_{t}\right)
$$

Therefore, by a known theorem, the zero solution of (1.2) is asymptotically stable. The primary difficulty here is in the construction of $V$.

The proof using the contraction mapping principle is much more straightforward. Let $\psi:[-r, 0] \rightarrow \mathbb{R}$ be a continuous initial function. Let $\mathbb{M}$ be the complete metric space of continuous functions $\phi:[-r, \infty) \rightarrow \mathbb{R}$ with $\phi_{0}=\psi$, and $\phi(t) \rightarrow 0$ as $t \rightarrow \infty$. By the variation of parameters formula, (1.2) is equivalent to

$$
x(t)=\psi(0) e^{-a t}+\int_{0}^{t} e^{-a(t-s)} b x(s-r) d s
$$

Define $P: \mathbb{M} \rightarrow \mathbb{M}$ by

$$
(P \phi)(t)=\psi(0) e^{-a t}+\int_{0}^{t} e^{-a(t-s)} b \phi(s-r) d s
$$

It is an easy exercise to show that $P$ is a contraction, and hence it has a unique fixed point $x$ which is also the solution of (1.2), with $x(t) \rightarrow 0$ as $t \rightarrow \infty$. This completes the proof that the zero solution of (1.2) is asymptotically stable. Notice that there is no need to construct a Liapunov function to show asymptotic stability of solutions of (1.2). 
Definition 1.1. A function $x$ is said to be an asymptotically stable solution of equation (1.1) if for every $\epsilon>0$, there exists a $T=T(\epsilon)$ such that for every $t \geq T$ and for every other solution $y$ of (1.1), $|x(t)-y(t)| \leq \epsilon$.

Our primary goal is to study the existence of asymptotically stable solutions of (1.1) defined in the above definition.

\section{PRELIMINARIES}

We employ a fixed point theorem due to Krasnosel'skii, which is stated below.

Theorem 2.1 (Krasnosel'skii, [10]). Let $\mathbb{M}$ be a closed convex nonempty subset of a Banach space $\mathcal{B}$. Suppose that $A$ and $B$ map $\mathbb{M}$ into $\mathbb{B}$ such that

(i) $x, y \in \mathbb{M}$ implies $A x+B y \in \mathbb{M}$;

(ii) $A$ is a contraction mapping; and

(iii) $B$ is a compact and continuous mapping.

Then there exists a $z \in \mathbb{M}$ with $z=A z+B z$.

We will use the Banach space

$$
B C:=\left\{x: \mathbb{R}_{+} \rightarrow \mathbb{R}^{n}: x \text { is bounded and continuous }\right\}
$$

with the norm $\|x\|=\sup _{t \geq 0}|x(t)|$ where $|\cdot|$ is a vector norm in $\mathbb{R}^{n}$.

Fixed point theorems, including Theorem 2.1, normally involve a compact mapping. For problems on finite intervals, this compactness is usually obtained by the Arzelà-Ascoli theorem. In the study of asymptotic stability by fixed point theory, it has been recognized that compactness on infinite interval presents problem. Banaś and Rzepka [2] studied the asymptotic stability of (1.1) employing a fixed point theorem of Darbo type associated with measures of noncompactness. Burton and Zheng [4] avoided the measures of noncompactness and studied the asymptotic stability using Krasnosel'skii's theorem under relatively weaker conditions. They obtained the compactness using a Arzelà-Ascoli type argument. Avramescu and Vladimirescu [1] employed Schauder's fixed point theorem and obtained the compactness using a 
different method. They obtained the asymptotic stability of solutions of the integral equation

$$
x(t)=q_{2}(t)+\int_{0}^{t} a(t) b(s) g(s, x(s)) d s .
$$

Our work of the present paper extends the work of [1]. We replace $q_{2}(t)$ and $a(t) b(s)$ by more general functions $f(t, x(t))$ and $K(t, s)$, respectively. In [1], the authors employed Schauder's fixed point theorem, whereas we employ Theorem 2.1, Krasnosel'skii's theorem. For the compactness of the mapping of $B$ of Theorem 2.1, we have adopted a technique found in [1].

Define the space $C_{l} \subset B C$ by

$$
C_{l}:=\left\{x \in B C: \lim _{t \rightarrow \infty} x(t) \in \mathbb{R}^{n} \text { exists }\right\} .
$$

Definition 2.2. A family $\mathcal{A} \subset C_{l}$ is called equiconvergent if for every $\epsilon>$ 0 , there exists a $T(\epsilon)>0$ such that for all $x \in \mathcal{A}$ and for all $t_{1}, t_{2} \geq T$, $\left|x\left(t_{1}\right)-x\left(t_{2}\right)\right| \leq \epsilon$.

On the space $C_{l}$, the following compactness criterion holds (see [1]).

Lemma 2.3. A family $\mathcal{A} \subset C_{l}$ is relatively compact if and only if

(a) $\mathcal{A}$ is uniformly bounded;

(b) $\mathcal{A}$ is equicontinuous on compact subsets of $\mathbb{R}_{+}$; and

(c) $\mathcal{A}$ is equiconvergent.

We will use this criterion for compactness when employing Theorem 2.1.

\section{ASYMPTOTICALLY STABLE SOLUTIONS}

In the following lemma we state certain assumptions on the kernel function $K$ of (1.1). The proof can be found in [1].

Lemma 3.1. Suppose the following hypotheses hold:

(H1) there exists an $M>0$ such that

$$
\int_{0}^{t}|K(t, s)| d s \leq M \text { for all } t \in \mathbb{R}_{+}
$$


(H2) for all $T>0$,

$$
\lim _{t \rightarrow \infty} \int_{0}^{T} K(t, s) d s=O_{n \times n}
$$

and

(H3)

$$
\lim _{t \rightarrow \infty} \int_{0}^{t} K(t, s) d s=I_{n \times n} .
$$

Then for every $x \in C_{l}$,

$$
\lim _{t \rightarrow \infty} \int_{0}^{t} K(t, s) x(s) d s=\lim _{t \rightarrow \infty} x(t) .
$$

Let $\rho>0$ be a constant, and define $B_{\rho}:=\left\{x \in \mathbb{R}^{n}:|x| \leq \rho\right\}$. Define

$$
m_{\rho}:=\sup \left\{|g(t, x)|: t \in \mathbb{R}_{+}, x \in B_{\rho}\right\} \text {, }
$$

and

$$
n_{\rho}:=\sup \left\{|f(t, x)|: t \in \mathbb{R}_{+}, x \in B_{\rho}\right\} .
$$

We assume $m_{\rho}<\infty$ and $n_{\rho}<\infty$.

The following assumptions will also be needed.

(A1) For all $x, y \in B_{\rho},|f(t, x)-f(t, y)| \leq \gamma(t)|x-y|$ for all $t \in \mathbb{R}_{+}$, where $\gamma(t) \leq \bar{\gamma}<1$ is continuous;

(A2) there exists a continuous function $\psi: B_{\rho} \rightarrow \mathbb{R}^{n}$ such that

$$
\lim _{t \rightarrow \infty} f(t, x)=\psi(x)
$$

(A3) there exists a $\theta \in \mathbb{R}^{n}$ such that

$$
\lim _{t \rightarrow \infty} g(t, x)=\theta,
$$

uniformly with respect to $x \in B_{\rho}$;

(A4)

$$
\lim _{t \rightarrow \infty} \int_{0}^{t}|K(t, s)| \phi(s) d s=0
$$

where $\phi(t)=\sup \left\{|g(t, x)-\theta|: x \in B_{\rho}\right\}$ for all $t \in \mathbb{R}_{+} ;$and 
(A5)

$$
\int_{0}^{t_{1}}\left|K\left(t_{1}, s\right)-K\left(t_{2}, s\right)\right| d s \rightarrow 0
$$

as $\left|t_{1}-t_{2}\right| \rightarrow 0$.

Choose a $\rho>0$ such that $n_{\rho}+M m_{\rho} \leq \rho$ for all $t \in \mathbb{R}_{+}$. Define $S_{\rho}=\{x \in$ $\left.C_{l}:\|x\| \leq \rho\right\}$. Clearly the set $S_{\rho}$ is a closed, bounded and convex subset of the Banach space $B C$.

Theorem 3.2. If assumptions (A1)-(A5) hold and if $K(t, s)$ satisfies the assumptions of Lemma 3.1, then (1.1) admits solutions in $S_{\rho}$, and every solution of this equation in $S_{\rho}$ is asymptotically stable.

Proof. For $x \in S_{\rho}$, define the operator $A$ by

$$
A x(t)=f(t, x(t))
$$

and define the operator $B$ by

$$
B x(t)=\int_{0}^{t} K(t, s) g(s, x(s)) d s .
$$

Let $x, y \in S_{\rho}$. Since $x \in S_{\rho}$, there exists an $x^{*} \in B_{\rho}$ such that $\lim _{t \rightarrow \infty} x(t)=$ $x^{*} \in B_{\rho}$. By (A2), since $\psi$ is continuous, $\lim _{t \rightarrow \infty} f(t, x(t))=\psi\left(x^{*}\right)$. By assumption (A3), we have that for all $y \in S_{\rho}$,

$$
\lim _{t \rightarrow \infty} g(t, y)=\theta
$$

The function $K(t, s)$ satisfies the hypotheses of Lemma 3.1. So,

$$
\lim _{t \rightarrow \infty} \int_{0}^{t} K(t, s) g(s, y(s)) d s=\theta .
$$

Thus $\lim _{t \rightarrow \infty}(A x+B y)(t)=\psi\left(x^{*}\right)+\theta$. Clearly $(A x+B y)(t)$ is continuous in t. Therefore, $A x+B y \in C_{l}$. In addition,

$$
\begin{aligned}
|(A x+B y)(t)| & \leq|f(t, x(t))|+\int_{0}^{t}|K(t, s)||g(s, y(s))| d s \\
& \leq n_{\rho}+M m_{\rho} \leq \rho .
\end{aligned}
$$

So, $A x+B y \in S_{\rho}$. Thus (i) from Theorem 2.1 holds. 
Next, notice assumption (A1) directly implies that $A$ is a contraction mapping. So (ii) from Theorem 2.1 holds.

Finally, we show (iii) of Theorem 2.1 holds. Define the operators: $U$ : $S_{\rho} \rightarrow C_{l}, G: S_{\rho} \rightarrow C_{l}$, by

$$
(U x)(t)=\int_{0}^{t} K(t, s) x(s) d s,
$$

and

$$
(G x)(t)=g(t, x(t))
$$

for $x \in S_{\rho}$, for all $t \in \mathbb{R}_{+}$. By Lemma 3.1, $U$ maps $S_{\rho}$ into $C_{l}$. By assumption (A3), $G$ maps $S_{\rho}$ into $C_{l}$. From the continuity of $g$, it follows that $G$ is continuous as a map. Also, since $U$ is linear, $U$ is continuous as a map. The continuity of $B$ follows immediately since $B x=(U \circ G) x$ for all $x \in S_{\rho}$.

Next, we use Lemma 2.3 to show $B S_{\rho}$ is relatively compact. First, notice for $x \in S_{\rho},|B x(t)| \leq M m_{\rho}$. So $B S_{\rho} \subset S_{\rho}$, showing that $B S_{\rho}$ is uniformly bounded.

Here we show $B S_{\rho}$ is equicontinuous on compact subsets of $\mathbb{R}_{+}$. Let $\gamma>0$ and $\epsilon>0$ be given. Then, for $x \in S_{\rho}$ and for $t_{1}, t_{2} \in[0, \gamma]$,

$$
\begin{aligned}
\left|(B x)\left(t_{2}\right)-(B x)\left(t_{1}\right)\right|= & \left|\int_{0}^{t_{2}} K\left(t_{2}, s\right) g(s, x(s)) d s-\int_{0}^{t_{1}} K\left(t_{1}, s\right) g(s, x(s)) d s\right| \\
& +\int_{0}^{t_{1}}\left|K\left(t_{2}, s\right)-K\left(t_{1}, s\right)\right||g(s, x(s))| d s \\
& +\int_{t_{1}}^{t_{2}}\left|K\left(t_{2}, s\right)\right||g(s, x(s))| d s .
\end{aligned}
$$

Let $\bar{K}=\max \left\{\left|K\left(t_{2}, s\right)\right|: s \in\left[t_{1}, t_{2}\right]\right\}$. For $\delta_{1}<\frac{\epsilon}{2 \bar{K} m_{\rho}}$, if $\left|t_{2}-t_{1}\right|<\delta_{1}$,

$$
\int_{t_{1}}^{t_{2}}\left|K\left(t_{2}, s\right)\right||g(s, x(s))| d s<\frac{\epsilon}{2} .
$$

Finally, by assumption (A5), there exists a $\delta_{2}>0$ such that for all $\left|t_{2}-t_{1}\right|<\delta_{2}$,

$$
\int_{0}^{t_{1}}\left|K\left(t_{1}, s\right)-K\left(t_{2}, s\right)\right| d s<\frac{\epsilon}{2 m_{\rho}} .
$$

This implies

$$
\int_{0}^{t_{1}}\left|K\left(t_{2}, s\right)-K\left(t_{1}, s\right)\right||g(s, x(s))| d s<\frac{\epsilon}{2}
$$


Let $\delta=\min \left\{\delta_{1}, \delta_{2}\right\}$. Then if $\left|t_{2}-t_{1}\right|<\delta$,

$$
\left|(B x)\left(t_{2}\right)-(B x)\left(t_{1}\right)\right|<\epsilon .
$$

So $B S_{\rho}$ is equicontinuous on compact subsets of $\mathbb{R}_{+}$.

Last, we show $B S_{\rho}$ is equiconvergent. Above it was shown that

$$
\lim _{t \rightarrow \infty} \int_{0}^{t} K(t, s) g(s, x(s)) d s=\theta
$$

By (A3), this convergence is uniform with respect to $x \in S_{\rho}$. So

$$
\lim _{t \rightarrow \infty}(B x)(t)=\theta
$$

uniformly with respect to $x \in S_{\rho}$. This implies $B S_{\rho}$ is equiconvergent.

Therefore, by Lemma 2.3, the set $B S_{\rho}$ is relatively compact. This implies the mapping $B: S_{\rho} \rightarrow S_{\rho}$ is compact. Since $B$ is continuous, (iii) from Theorem 2.1 holds.

Thus, by Theorem 2.1, there exists an $x \in S_{\rho}$ such that $x=A x+B x$. By the construction of $A$ and $B$, this $x$ is a solution of (1.1).

Now, let $x_{1}, x_{2} \in S_{\rho}$ be two solutions of (1.1). Since $x_{1}(t)=\left((A+B) x_{1}\right)(t)$, and $x_{2}(t)=\left((A+B) x_{2}\right)(t)$ for all $t \in \mathbb{R}_{+}$, it follows from (A1) and (A4) that

$$
\begin{aligned}
\left|x_{1}(t)-x_{2}(t)\right| \leq & \gamma(t)\left|x_{1}(t)-x_{2}(t)\right|+\left|\int_{0}^{t}\right| K(t, s)\left|\left[g\left(s, x_{1}(s)\right)-\theta\right]\right| d s \\
& +\int_{0}^{t}|K(t, s)|\left[g\left(s, x_{2}(s)\right)-\theta\right] \mid d s \\
\leq & \gamma(t)\left|x_{1}(t)-x_{2}(t)\right|+2 \int_{0}^{t}|K(t, s)| \phi(s) d s .
\end{aligned}
$$

Therefore, for all $t \in \mathbb{R}_{+}$,

$$
(1-\gamma(t))\left|x_{1}(t)-x_{2}(t)\right| \leq 2 \int_{0}^{t}|K(t, s)| \phi(s) d s
$$

which implies

$$
\left|x_{1}(t)-x_{2}(t)\right| \leq \frac{2}{1-\gamma(t)} \int_{0}^{t}|K(t, s)| \phi(s) d s .
$$

Since $\gamma(t) \leq \bar{\gamma}<1$, there exists a positive constant $L$ such that $\frac{2}{1-\gamma(t)} \leq$ $\frac{2}{1-\bar{\gamma}}:=L$. Now, from (A4), it follows that there exists a $T$ such that for any $\epsilon>0$,

$$
\int_{0}^{t}|K(t, s)| \phi(s) d s \leq \frac{\epsilon}{L}
$$


for $t \geq T$. Then for all $t \geq T$,

$$
\begin{aligned}
\left|x_{1}(t)-x_{2}(t)\right| & \leq L \int_{0}^{t}|K(t, s)| \phi(s) d s \\
& \leq L \frac{\epsilon}{L}=\epsilon .
\end{aligned}
$$

This shows that every solution of (1.1) in $S_{\rho}$ is asymptotically stable, which concludes the proof.

\section{AN EXAMPLE}

Consider the scalar nonlinear Volterra integral equation

$$
x(t)=\frac{\beta x(t)}{\left(1+2[x(t)]^{2}\right)}+\int_{0}^{t} e^{-(t-s)}\left(\frac{|x(s)|}{1+[x(s)]^{2}} e^{-s}+\theta\right) d s,
$$

where $\beta<1$ and $\theta$ is any real number. Then functions $f, g$ and $K$ satisfy all assumptions in Theorem 3.2. Notice $m_{\rho} \leq 1+\theta, n_{\rho} \leq \beta$, and $M=1$. So by Theorem 3.2, solutions of (4.1) in $S_{\rho}$, for $\rho \geq \beta+\theta+1$, are asymptotically stable.

\section{REFERENCES}

[1] C. Avramescu, C. Vladimirescu, On the existence of asymptotically stable solution of certain integral equations, Nonliner Anal. 66 (2007), 472-483.

[2] J. Banaś, B. Rzepka, An application of a measure of noncompactness in the study of asymptotic stability, Appl. Math. Lett. 16 (2003), 1-6.

[3] L. C. Becker, T. A. Burton, Stability, fixed points and inverses of delays, Proc. Roy. Soc. Edinburgh Sect. A 136 (2006), 245-275.

[4] T.A. Burton, B. Zhang, Fixed points and stability of an integral equation: nonuniqueness, Appl. Math. Lett. 17 (2004), 839-846.

[5] T.A. Burton, Stability by fixed point theory for functional differential equations, Dover Publications INC. Mineola, New York, 2006. 
[6] Y. Hino, S. Murakami, Stabilities in linear integrodifferential equations, Lecture Notes Numer. Appl. Anal. 15 (1996), 31-46.

[7] T. Furumochi, S. Murakami, Y. Nagabuchi, Stabilities in Volterra difference equations on a Banach space, in: Differences and differential equations, Amer. Math. Soc., Providence, RI, (2004), 159-175.

[8] U. Mehmet, Y. Raffoul, Stability in nonlinear delay Volterra integrodifferential systems, J. Nonlinear Sci. 7 (2014), 422-428.

[9] J. LaSalle, S. Lefschetz, Stability by Liapunov's direct method, with applications, Academic Press, New York, 1961.

[10] D. R. Smart, Fixed point theorems, Cambridge University Press, LondonNew York, 1974.

[11] Tingxiu Wang, Exponential stability and inequalities of solutions of abstract functional differential equations, J. Math. Anal. Appl. 324 (2006), 982-991.

[12] T. Yoshizawa, Stability theory by Liapunov's second method, Mathematical Society of Japan, Tokyo, 1966.

[13] B. Zhang, Asymptotic stability in functional-differential equations by Liapunov functionals, Trans. Amer. Math. Soc. 347 (1995), 1375-1382. 
